JOURNAL OF

APPLIED

CRYSTALLOGRAPHY

ISSN 1600-5767

\section{Neutron Interferometry: Lessons in Experimental Quantum Mechanics, Wave-Particle Duality, and Entanglement. 2nd Ed. By Helmut Rauch and Samuel A. Werner. Oxford University Press, 2015. Price (hardcover) GBP 70. ISBN 978-0-19-871251-0.}

\author{
Mark P. Silverman*
}

Received 17 April 2015
Accepted 1 August 2015

Edited by M. Nespolo, Université de Lorraine, France

Keywords: book reviews; neutron interferometry.

Mark P. Silverman (http://www.trincoll.edu/ silverma) is the G. A. Jarvis Professor of Physics at Trinity College. His most recent books discussing his research in quantum physics, nuclear physics and statistical physics are $A$ Certain Uncertainty: Nature's Random Ways (Cambridge University Press, 2014) and And Yet It Moves: Strange Systems and Subtle Questions in Physics, 2nd ed. (Cambridge University Press, 2015).

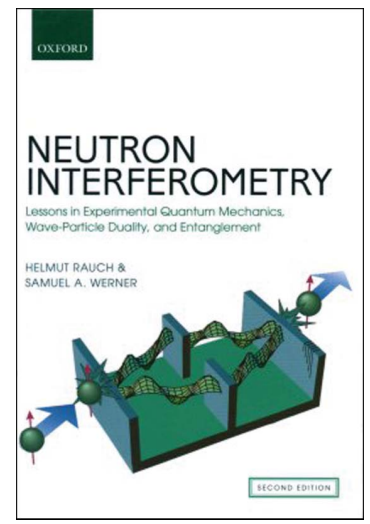

(C) 2015 International Union of Crystallography
Trinity College, 300 Summit Street, Hartford, CT 06106, USA. *Correspondence e-mail: mark.silverman@trincoll.edu

There is a story, perhaps apocryphal, that Nobel Laureate physicist I. I. Rabi, upon learning of the discovery of the muon in 1936, exclaimed 'Who ordered that!' His surprise came from the belief that, with the discovery of the neutron by James Chadwick just four years earlier, physicists' model of the sub-atomic world was satisfactorily complete. At the time of its discovery in 1932, the neutron was precisely what physicists would have ordered to resolve a variety of troubling observations. It accounted for the existence of isotopes, for the neutral non-electromagnetic radiation that emerged from alpha particle bombardment of certain elements like beryllium or boron, for the apparent emergence of electrons from inside a nucleus that underwent beta decay, and for the puzzling fact that nitrogen-14 was a boson when, according to a proton-electron model, it should have been a fermion.

However satisfied Chadwick may have been to find that the neutron resolved a host of problems physicists faced in the 1930s, were he alive now I suspect he would be rendered speechless in amazement by Rauch and Werner's comprehensive and lucid book of the very many ways neutrons have been employed since then to test physical principles and to study the properties of condensed matter. At the core of this versatility is the fact that the neutron participates in all the known fundamental interactions: the strong nuclear interaction (it helps maintain the integrity of a nucleus against the mutual electrostatic repulsion of protons), the weak nuclear interaction (it is itself unstable against beta decay with a free-state half-life of about $10 \mathrm{~min}$ ), gravity (it not only falls in a gravitational field but responds to a gravitational potential under conditions where there is no classical gravitational force) and electromagnetism (although it is neutral, it has a magnetic moment and therefore can respond to magnetic fields as well as to laboratory electric fields that, by virtue of special relativity, transform to magnetic fields in the neutron's rest frame).

Neutron Interferometry examines the manifold consequences of these interactions through detailed explanations of experiments, executed for the most part within the authors' own research groups, employing the extraordinarily sensitive perfect silicon crystal neutron interferometer. Although a wide variety of experiments are described in the book, the typical experimental configuration is that of a Mach-Zehnder interferometer of monolithic design, the various reflecting plates of which were cut from a single Si crystal which forms the base. The essential feature of the apparatus, as highlighted by the authors, is that the reflecting planes are arranged undisturbed throughout the entire macroscopic apparatus (enclosed areas up to about $100 \mathrm{~cm}^{2}$ have been tested) with a precision comparable to the lattice spacing.

Although a prospective reader may infer from the book title that the subject is limited to interferometry, one discovers immediately from perusing the contents that, from the perspective of modern physics, this is hardly a limitation at all. Interferometry, whether optical, electron or neutron, has played a key role in elucidating many of the most fundamental physical problems of the 20th century, and I expect that to continue throughout the 21st. Among the numerous issues upon which neutron interferometry has been brought to bear, as discussed by Rauch and Werner, are key questions relating to 
(a) symmetry (neutrons are spin-1/2 particles and therefore have two spin states that are similar in some ways and very different in others from light, which also has two independent states of polarization);

(b) geometry and topology (neutrons exhibit a waveparticle duality ideal for testing phase-sensitive effects arising from unusual configurations of classical fields and potentials);

(c) inertia and motion (neutron interferometry, like laser interferometry, is very sensitive to rotational motion and other forms of acceleration);

(d) applications to solid state physics (neutrons are highly penetrating and provide information about the magnetic structure of their environment through their scattering or diffraction).

In addition to the wide range of phenomena that have been investigated with neutrons, Rauch and Werner speculate in a chapter of multiple sections on the possibilities of using neutron interferometry to look for new quantum effects. Among these especially challenging experiments are proposals to

(a) test the Schrödinger equation for a nonlinear contribution of the wavefunction;

(b) look for neutron-antineutron oscillations (in analogy to already observed oscillations between neutrino types);

(c) search for quantum entanglement of adjacent protons or deuterons;

(d) observe quantum effects in neutron scattering analogous to the Hanbury Brown-Twiss (HBT) effects with incoherent light.

As an atomic and nuclear physicist myself, who has investigated - albeit with electrons, rather than neutrons - the possibilities of a 'quantum optics of particles' (Silverman, 2008), I found the discussion of the last topic particularly interesting and worthy of a brief elaboration here.

HBT first employed intensity interferometry, as they called their method of measurement, to view an incoherent light source (a star) with two detectors whose outputs were correlated. From the variation in signal with increasing path length difference or delay time between the detectors, HBT were able to determine the diameter of the star. What astonished many physicists around the time (mid-1950s) of HBT's experiments was that the method yielded a kind of interference pattern despite the fact that the delay time between the two detectors was vastly greater than the coherence time of the source. Eventually it was demonstrated that the signal rendered by an intensity interferometer is not really an interference of intensities, but a measure of the second-order coherence of the source, in contrast to standard interferometry which is sensitive to first-order coherence.
A characteristic feature of second-order coherence is that it depends on the degeneracy of the source, i.e. the mean number of quanta per cell of phase space. In this regard, intensity interferometry is sensitive to the fermionic or bosonic nature of the particles of the source. In the 1980s I proposed second-order interferometric experiments for massive particles, rather than for light, to look for novel, purely quantum effects relating to quantum statistics (e.g. particle bunching, anti-bunching and other effects) and forcefree potential fields (e.g. phenomena dependent on electric charge like the Aharonov-Bohm effect or on particle mass like the Colella-Overhauser-Werner effect). Theoretical analyses indicated that such experiments are feasible with a field-emission electron beam, but that the degeneracy of extant neutron sources was orders of magnitude too low. Indeed, the first successful observation of electron antibunching was made in 2002 with a low-voltage field-emission beam. However, as Rauch and Werner describe, free-fermion anti-bunching was observed with thermal neutrons not long thereafter in 2006, an achievement made possible by new advanced instrumentation, careful experimental design and precise knowledge of the statistics of the source (Iannuzzi et al., 2006).

The second edition of Neutron Interferometry (2015) extends the first edition (2000) by a new chapter on 'quantum contextuality', a term that refers to the effect on a measurement of the experimental arrangement of some other previous or simultaneous measurement. The original chapters have also been expanded in places so that the new edition is longer than the first by about 50 pages. Although a search for books about neutrons will bring up a substantial number, these others are almost all about nuclear reactions and power reactors. There is, of course, the invaluable encyclopedic treatise by James Byrne (2011), but the chapter on neutron wave phenomena in that book takes up a mere 79 out of 760 pages. In short, Rauch and Werner 's Neutron Interferometry is a unique work, carefully organized and clearly written, that informs the reader of the fascinating universe of quantum phenomena revealed by the wave-like behaviour of the neutron. It is also a testament to the remarkable experimental achievements in the long productive careers of the two authors.

\section{References}

Byrne, J. (2011). Neutrons, Nuclei \& Matter: An Exploration of the Physics of Slow Neutrons. Mineola: Dover.

Iannuzzi, M., Orecchini, A., Sacchetti, F., Facchi, P. \& Pascazio, S. (2006). Phys. Rev. Lett. 96, 080402.

Silverman, M. P. (2008). Quantum Superposition: Counterintuitive Consequences of Coherence, Entanglement, and Interference. Heidelberg: Springer. 